\title{
Probabilistic Two-Phase Aircraft Wake-Vortex Model: Further Development and Assessment
}

\author{
Frank Holzäpfel* \\ DLR, German Aerospace Research Center, Oberpfaffenhofen, 82234 Weßling, Germany
}

\begin{abstract}
Further developments, applications, and assessments of the probabilistic two-phase aircraft wake-vortex model P2P are described. The wake-vortex model is applied to data of two field measurement campaigns accomplished at Tarbes airport, France. Measurements corroborate unambiguously the two-phase circulation decay anticipated by theory and parameterized by P2P. Vortex age and descent speed are adjusted to match effects of axial wind and glide-slope angle. Envelopes of vortex trajectories are expanded to consider tilting, stalling, and rebounding wake vortices caused by axial- and crosswind shear. For probabilistic model output a choice between arbitrary degrees of probability is established, and a stochastic prediction mode is introduced. In a deterministic scoring procedure, model perfomance is compared to the skill of another model. Probabilistic model performance is evaluated by the compilation of probability density distributions that relate wake vortex measurement data to the predicted envelopes.
\end{abstract}

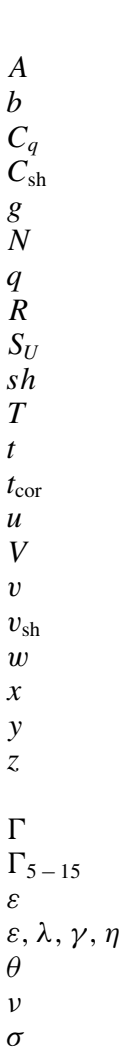

Subscripts

$l \quad=$ lower limit

meas $\quad=$ measured

prob $\quad=$ selected probability

$u \quad=$ upper limit

Received 24 March 2005; revision received 28 June 2005; accepted for publication 28 June 2005. Copyright (C) 2005 by the American Institute of Aeronautics and Astronautics, Inc. All rights reserved. Copies of this paper may be made for personal or internal use, on condition that the copier pay the $\$ 10.00$ per-copy fee to the Copyright Clearance Center, Inc., 222 Rosewood Drive, Danvers, MA 01923; include the code 0021-8669/06 \$10.00 in correspondence with the CCC.

*Research Scientist, Institut für Physik der Atmosphäre; frank. holzaepfel@dlr.de.

$$
\begin{array}{ll}
0 & =\text { initial value } \\
1 & =\text { first decay phase } \\
2 & =\text { second decay phase }
\end{array}
$$

Superscripts

* $\quad=$ normalized by initial vortex parameters $b_{0}, t_{0}, w_{0}, \Gamma_{0}$

= normalized by probabilistic bounds

\section{Introduction}

C OUNTER-ROTATING aircraft trailing vortices are generated $\checkmark$ as a natural consequence of lift. Depending on meteorological conditions, these wake vortices can persist for several minutes and, therefore, can pose a potential risk to aircraft following behind. To avoid wake-vortex encounters, aviation authorities established separation standards between consecutive aircraft. Currently effective separation standards can heavily degrade aviation efficiency when traffic congestion limits airport capacity during landing and takeoff. A system that would allow the relaxation of current aircraft separations under favorable weather conditions, while keeping safety at least at the same level, could provide significant economizations. ${ }^{1,2}$ An overview on the state of the art and the components of envisaged wake-vortex advisory systems is given in Ref. 3 .

A key element of such a wake-vortex advisory system is a parametric model that reliably predicts wake-vortex evolution along the flight path in real time. The model should consider the effects of aircraft configuration, wind, wind shear, turbulence, temperature stratification, and proximity of the ground. To account for the stochastic characteristics of wake-vortex behavior, the model should not be restricted to deterministic predictions. Quite the contrary, it should provide probabilistic predictions that are translated into envelopes for vortex trajectories and strengths combined with clearly specified probabilities. From the number of suggested wake-vortex models (see Ref. 4 for a list of models), only few comply with most of the listed requirements. In recent years, mainly three models ${ }^{4-6}$ have been further developed and tested. ${ }^{7,8}$ Applications and an assessment of the probabilistic two-phase aircraft wake-vortex model ${ }^{4}$ (P2P) based on data of the field deployments performed at International Airports Memphis, Tennessee (December 1994, August 1995) and Dallas Fort Worth, Texas (September/October 1997), and the WakeOP campaign at the airfield in Oberpfaffenhofen, Germany (April/May 2001) are described in Ref. 9.

In the current manuscript, the P2P model formulation is briefly recapitulated, and the employed databases are introduced. Further developments of $\mathrm{P} 2 \mathrm{P}$ are described. Vortex age and descent speed are adjusted to match effects of axial wind and glide-slope angle. 
Probabilistic envelopes are expanded to consider tilting, stalling, and rebounding wake vortices caused by axial- and crosswind shear. Operational significance of disconnected long-lived vortex segments is discussed. Model validation is pursued based on data of two field campaigns accomplished at Tarbes Airport, France. The performance of a deterministic version of $\mathrm{P} 2 \mathrm{P}$ is compared to the skill of another model. ${ }^{8}$ Probabilistic model performance is evaluated by the compilation of probability density distributions, which relate wake-vortex measurement data to the bounds of the predicted uncertainty allowances. Based on this approach, the predicted envelopes of vortex trajectories and circulation are adjustable to arbitrary degrees of probability. Finally, a stochastic prediction mode that can be employed in a Monte Carlo simulation environment is introduced.

\section{Probabilistic Two-Phase Aircraft Wake-Vortex Model}

A detailed description of P2P is given in Ref. 4. Here only main properties of that model version are briefly recapitulated. The original P2P model accounts for the effects of wind, turbulence, stable thermal stratification, and ground proximity. Input data that characterize the wake vortices are time of vortex generation, initial position, circulation, and vortex spacing. Environmental input parameters are vertical profiles of crosswind, rms value of ambient turbulence, eddy dissipation rate (EDR), and potential temperature. For the parameterizations of headwind and wind shear effects described in the current manuscript, the model requires additionally the axial wind profile, aircraft ground speed, and glide-slope angle.

The model is formulated in normalized form where the characteristic scales are based on initial vortex separation and circulation leading to the timescale $t_{0}=2 \pi b_{0}{ }^{2} / \Gamma_{0}$. EDR is normalized according to $\varepsilon^{*}=\left(\varepsilon b_{0}\right)^{1 / 3} / w_{0}$, where $w_{0}=\Gamma_{0} / 2 \pi b_{0}$ denotes the initial descent speed, and temperature stratification is expressed by the normalized Brunt-Väisälä frequency $N^{*}=\left(g / \theta_{0} \mathrm{~d} \theta / \mathrm{d} z\right)^{1 / 2} t_{0}$. P2P employs a circulation $\Gamma_{5-15}^{*}$, which is averaged over circles with radii from 5 to $15 \mathrm{~m}$. Benefits and drawbacks of radii-averaged circulation definitions are discussed in Ref. 10.

For the prediction of circulation, the concept of two-phase circulation decay is pursued (see Fig. 1). For comparison, predictions of the AVOSS Prediction Algorithm (APA $)^{8}$ are included in the figure denoted by gray dashed lines. The turbulent diffusion phase described by part 1 of Eq. (1) is followed by a rapid decay phase that can be parameterized by the full equation

$$
\Gamma_{5-15}^{*}\left(t^{*}\right)=\underbrace{A-\exp \frac{-R^{2}}{v_{1}^{*}\left(t^{*}-T_{1}^{*}\right)}}_{1}-\exp \frac{-R^{2}}{v_{2}^{*}\left(t^{*}-T_{2}^{*}\right)}
$$

The onset time of rapid decay at $T_{2}^{*}$ depends on ambient turbulence and stratification. Figure 2 depicts the dominant impact of turbulence on $T_{2}^{*}$. The respective decay rate is adjusted by the effective viscosity $v_{2}^{*}$, which mainly depends on ambient stratification. The constant parameters $T_{1}^{*}$ and $\nu_{1}^{*}$ control decay in the diffusion phase,
$R^{*}$ corresponds to a mean radius, and $A$ is a constant to adjust $\Gamma_{5-15}^{*}\left(t^{*}=0\right)$. The descent rate obeys a nonlinear dependence on circulation, which allows for a reduction of circulation without the reduction of the descent rate during the early vortex evolution and stagnating or even rebounding vortices with nonzero circulation in strongly stably stratified environments.

Precise deterministic wake-vortex predictions are not feasible operationally. Primarily, it is the nature of turbulence that deforms and transports the vortices in a stochastic way and leads to considerable spatiotemporal variations of vortex position and strength. Moreover, uncertainties of aircraft parameters and the strong temporal and spatial variability of environmental conditions must be taken into account. Therefore, $\mathrm{P} 2 \mathrm{P}$ is designed to predict wake-vortex behavior within defined confidence intervals. For this purpose, decay parameters $T_{2}^{*}$ and $v_{2}^{*}$ are varied in consecutive model runs, and various static and dynamic uncertainty allowances are added which consider the increased scatter in turbulent environments. A deterministic model version termed D2P provides mean wake-vortex evolutions employing intermediate decay parameters.

\section{Wake-Vortex Databases}

The wake-vortex forecasting and measurement campaigns WakeTOUL (May/June 2002) and Flight-Test 1 of AWIATOR (August 2003) both have been accomplished at Tarbes Airport, France, with partners from Airbus, ONERA, and DLR. WakeTOUL was part of DLR's "Projekt Wirbelschleppe" and was cofunded by the European Commission (EC) project C-Wake. AWIATOR is a multilateral

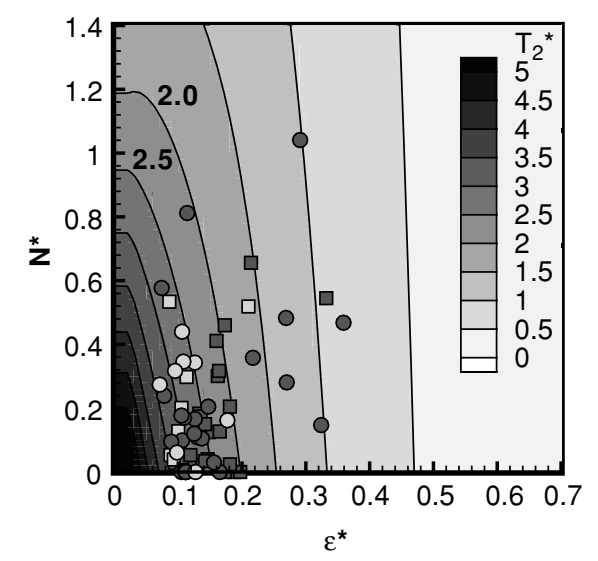

Fig. 2 Onset time of rapid decay as a function of mean normalized eddy dissipation rate and Brunt-Väisälä frequency: $\bigcirc$, WakeToul overflight conditions (32 cases); $\square$, conditions during AWIATOR-FT1 (32 cases). Dark-gray symbols distinguish data used for the establishment of scoring results in Table 1 and PDDs in Fig. 9 from the remainder, plotted in light-gray symbols.
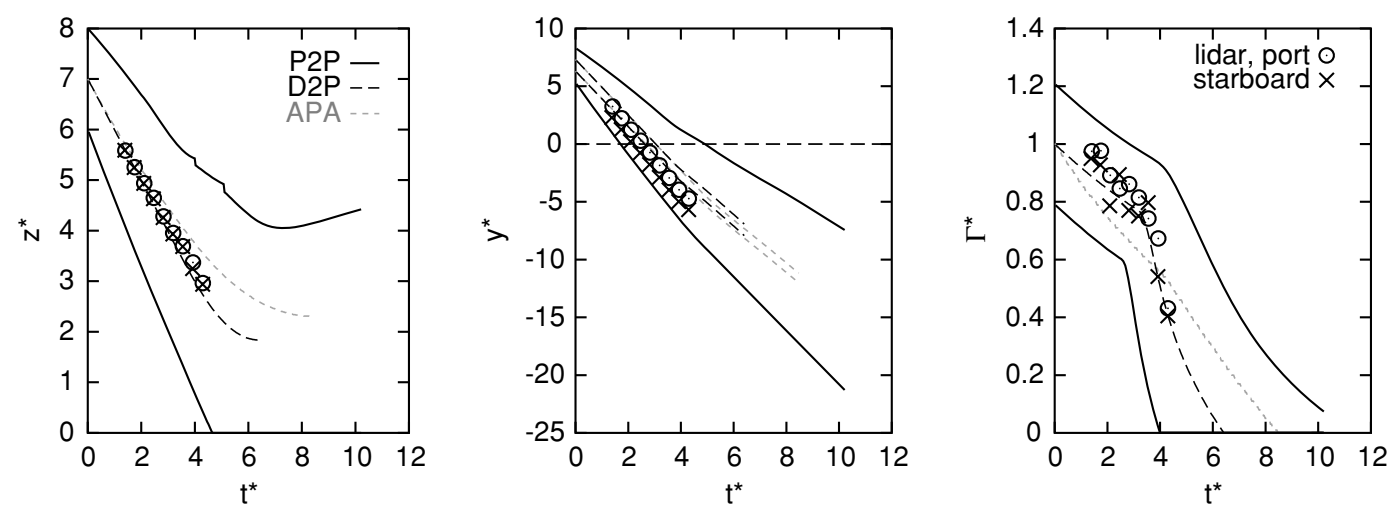

Fig. 1 Measured (symbols) and predicted (lines) evolution of normalized vertical and lateral positions and circulation of trailing vortices from WakeTOUL flight 4-17: - . - , deterministic behavior; _ _ , respective probabilistic envelope of P2P. Predictions of APA plotted in gray dashed lines. 
technology platform cofunded by the EC. The primary objective of the campaigns was to trace wake-vortex trajectories and circulation evolutions under calm and neutral atmospheric conditions with high accuracy and until a progressed state of vortex decay. A survey on the campaigns is given in Ref. 3. In the following only the subset of instrumentation used for the current investigations is described.

Wake vortices generated by a large transport aircraft, which performed overflights at constant heights ranging from $180 \mathrm{~m}$ to $400 \mathrm{~m}$, were traced with a $2-\mu \mathrm{m}$ pulsed lidar system. ${ }^{11}$ Time and position of the dedicated overflights were planned based on predictions of environmental parameters by the weather forecast model system NOWVIV $^{9}$ (nowcasting wake-vortex impact variables) and respective wake predictions with P2P. From each campaign 32 overflights are used for the current analysis. During eight of these overflights, glide-slope angles of 1.9 or $3.9 \mathrm{deg}$ were flown. The lidar scanned the measurement plane perpendicular to the direction of the approaching aircraft employing different elevation sectors within a range of 0 to $30 \mathrm{deg}$. For the evaluation of wake-vortex properties, an interactive four-stage data-processing algorithm, which is described in detail in Ref. 11, was applied to data covering distances from 500 to $1100 \mathrm{~m}$. Profiles of vortex tangential velocities were estimated with a vertical resolution of less than $2 \mathrm{~m}$. From these profiles vortex positions and circulations were derived. The error of circulation measurements was determined to $13 \mathrm{~m}^{2} / \mathrm{s}$ (see Ref. 12).

Five-minute averages of vertical profiles of crosswind and EDR were estimated from data in a 500- to $2400-\mathrm{m}$ range. ${ }^{13}$ For WakeToul, EDR was estimated from the difference of the velocity structure function determined over distances of 60 and $120 \mathrm{~m}$. For AWIATOR-FT1, EDR estimates derived from the structure function determined along distances of 90 and $180 \mathrm{~m}$ and from Doppler spectrum width are available additionally. P2P employs the minimum of EDR values achieved by the three different methods. Wake-vortex predictions employing EDR data based on the velocity structure function taking into account the outer scale of turbulence ${ }^{13}$ yield similar results but are not considered here.

A Sodar with a RASS extension provided vertical profiles of the three wind components, vertical fluctuation velocity, and virtual temperature. The vertical resolution was adjusted to $20 \mathrm{~m}$ and the averaging time to $10 \mathrm{mins}$. Based on the assumption of isotropy, the rms value of turbulence was calculated from the vertical fluctuation velocity. The Brunt-Väisälä frequency was derived from the virtual temperature profiles. The Sodar/RASS system was complemented by a sonic anemometer with a sampling frequency of $20 \mathrm{~Hz}$ mounted on a 10-m mast. By default wake-vortex predictions employ EDR data from lidar and wind, fluctuation velocity, and temperature data from the Sodar/RASS system and the sonic anemometer.

Figure 2 displays the distribution of mean values of normalized eddy dissipation rates and Brunt-Väisälä frequencies for each overflight. During WakeToul (AWIATOR-FT1), the mean eddy dissipation rate was $\overline{\varepsilon^{*}}=0.15(0.15)$ and varied between 0.071 $(0.086) \leq \varepsilon^{*} \leq 0.36(0.33)$. The mean Brunt-Väisälä frequency was $\overline{N^{*}}=0.24(0.18)$ within bounds of $0 \leq N^{*} \leq 1.04(0.66)$. On average, atmospheric conditions in the WakeToul and the AWIATORFT1 campaigns were quite similar. Both turbulence and temperature stratification levels represent a wide range from weak to strong conditions. Nevertheless, during a majority of overflights the objective to observe long-lived wake vortices under calm and weakly stratified conditions was achieved.

\section{Further Development}

Further developments of P2P regarding circulation decay, effects of axial wind and glide-slope angle, and axial- and crosswind shear are introduced in the following. New approaches concerning model validation and probabilistic and stochastic prediction modes are described in subsequent sections.

\section{Persistence}

With the field experiments WakeTOUL and AWIATOR-FT1 the superior suitability of the $2-\mu \mathrm{m}$ pulsed Doppler lidar for wake vortex characterization has been demonstrated. ${ }^{11}$ Two decisive factors,

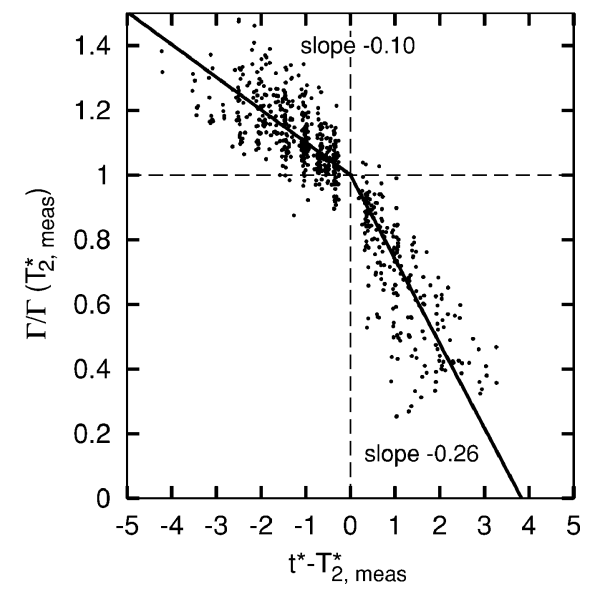

Fig. 3 Scatter plot of circulation evolutions measured by lidar during 48 overflights. Time coordinate shifted by observed onset time of rapid decay $T_{2 \text {,meas }}^{*}$ and circulation normalized by circulation measured at $t^{*}=T_{2, \text { meas }}^{*}$

the long-range capability of more than $1 \mathrm{~km}$ and a four-stage data processing, enable observations over periods from vortex generation to decay.

Figures 1 and 3 demonstrate that the two-phase decay anticipated by simulations and theory ${ }^{14-16}$ now for the first time could be unambiguously corroborated by measurements. The scatter plot of measured circulation data of 48 overflights depicted in Fig. 3 clearly supplies evidence of the previously controversly discussed two-phase vortex evolution. ${ }^{1,17}$ To clearly demonstrate two-phase decay characteristics, the time coordinate in Fig. 3 is shifted by the observed onset time of rapid decay $T_{2 \text {,meas }}^{*}$, and circulation is normalized by the respective circulation value for every individual overflight. The different slopes of the best-fit straight lines confirm the accelerated decay during late vortex evolution.

The long observation times achieved during WakeTOUL and AWIATOR-FT1 campaigns suggest increasing the onset time of rapid decay $T_{2}^{*}$ by a factor of 1.2 . This delayed decay approximately also covers prolongated lifetimes caused by constant background shear described in Ref. 9.

Nevertheless, a few cases were observed with apparently nonconservative P2P predictions. As demonstrated in Fig. 4, these cases at first typically develop almost deterministically. Then they are characterized by one or several time units without any lidar observation. The subsequent recurrence suggests that vortices are pinched off, and the remaining, often strongly deformed, vortex segments are advected through the measurement plane by axial winds. The measurements do not reveal whether the observed sequences denote the classical formation of long-lived vortex rings or rather the occurence of far-separated vortex segments for which mutual destruction mechanisms ${ }^{16}$ do not apply. Note that in Fig. 3 these lately recurred observations were omitted in order to avoid the concealment of the preceding two-phase characteristics of vortex decay.

Several factors suggest that the long-lived vortex segments do not constitute potentially hazardous situations. First, persistent but operationally insignificant vortex patches occur typically during turbulent vortex decay. Figure 5 illustrates the substantial spread of circulation values derived from a single large-eddy simulation of trailing vortex evolution in a turbulent atmosphere. ${ }^{15}$ Because the axial extension of the simulation is restricted, real trailing vortices might potentially exhibit an even larger spread. Second, flight simulator studies ${ }^{18}$ indicate significant reductions in the maximum bank angles experienced by encountering aircraft when vortices become wavy and break up into rings. Third, the fact that trailing vortices frequently persist longer than ICAO separations ${ }^{19}$ suggests that vortex deformation constitutes an important contribution to the high level of safety of current aircraft separations.

We conclude that the deformed vortex segments, whose circulation was undersestimated by $\mathrm{P} 2 \mathrm{P}$, most likey do not constitute a 

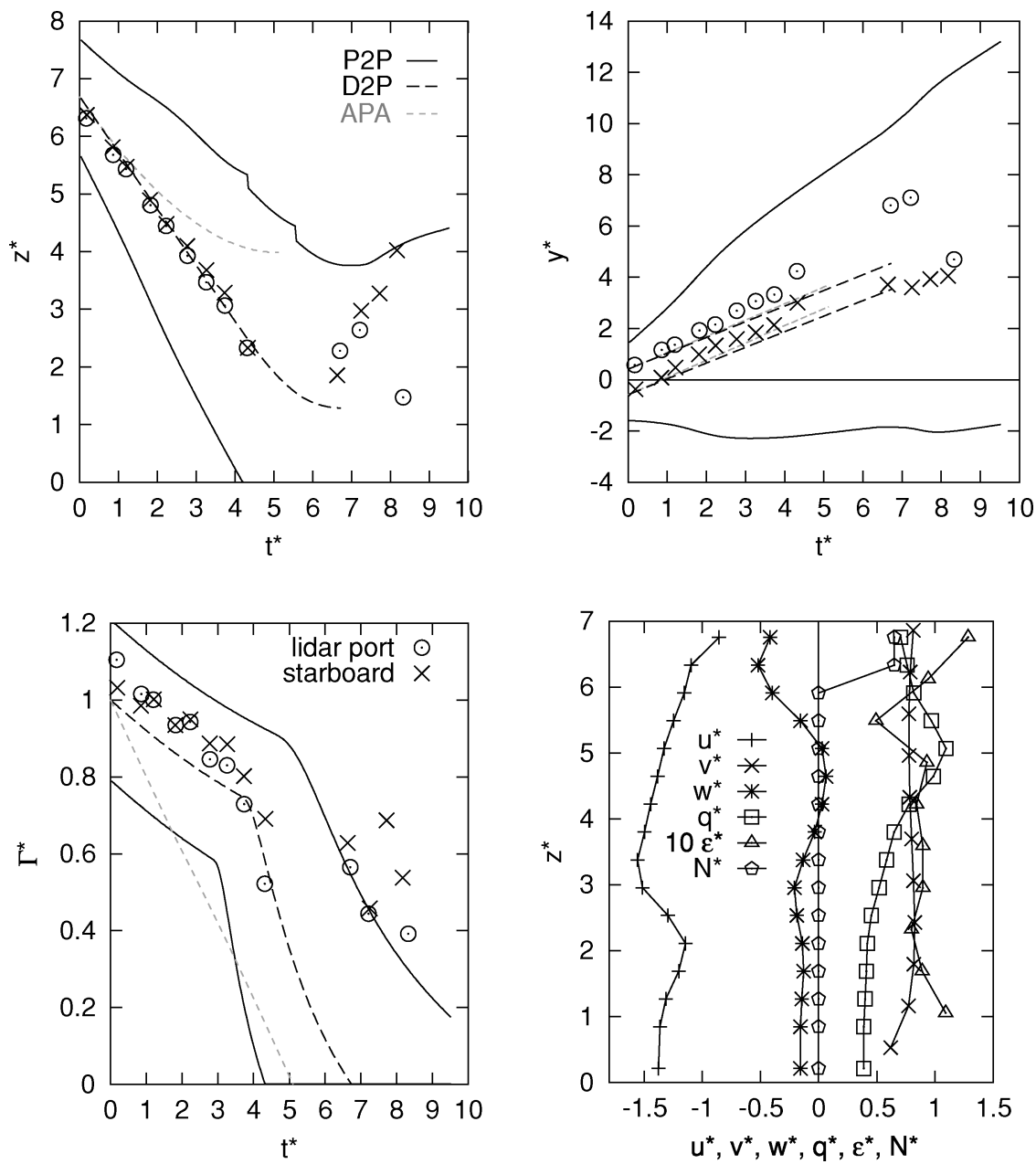

Fig. 4 Measured (symbols) and predicted (lines) evolution of trailing vortices from AWIATOR-FT1 flight 2-04 and vertical profiles of environmental data (crosswind provided by lidar).

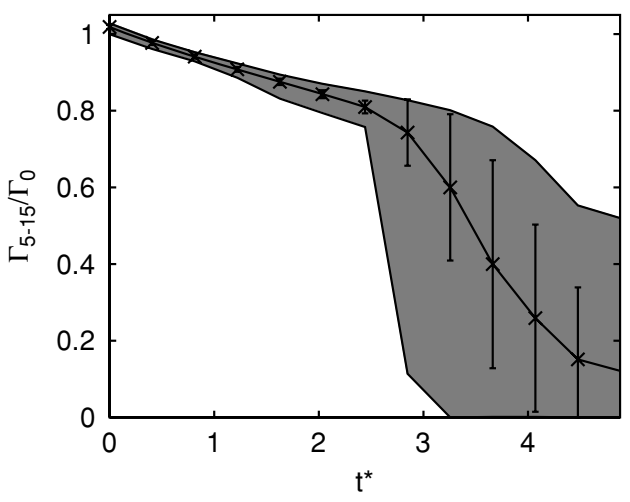

Fig. 5 Temporal evolution of radii-averaged circulation from LES of vortex evolution in turbulent atmosphere. ${ }^{15}$ Crosses denote average $\Gamma_{5-15} / \Gamma_{0}$, error bars are standard deviations, and shaded area covers the range between maximum and minimum values.

potential risk to follower aircraft because of their patchy structure and the corresponding strongly reduced exposure times. To take into account the observed effects, nevertheless, a real-time wakevortex model should possibly also predict vortex deformation and breakup. This, however, would complicate the definition of nonhazard criteria. For the moment, we rather prefer to conservatively reproduce punctiform lidar measurements with high confidence (see the following assessments) and keep the overestimation of potential hazards as an additional safety factor within a wake-vortex spacing system.

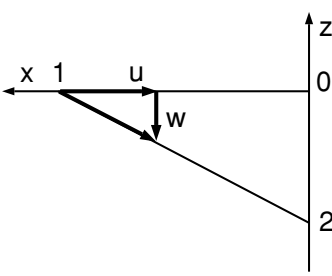

Fig. 6 Sketch illustrating vortex transport in a constant headwind situation at level flight.

Headwind

Compared to a calm situation, headwind (tailwind) advects younger (older) vortex segments into the lidar observation plane, which is usually spanned perpendicular to the flight direction and in which $\mathrm{P} 2 \mathrm{P}$ predicts vortex evolution. For headwind, the resulting difference in age $\Delta t$ corresponds to the time the aircraft needs to fly from the vortex prediction plane at $x=0$ (see position 0 in Fig. 6) to position 1 where the vortex segments are generated. Subsequently, the segments are advected into the vortex prediction plane at position 2. On their way into the prediction plane, the vortices follow a path defined by axial wind velocity $u$ and vortex descent speed $w$. The corresponding vortex age offset depends on axial wind velocity and aircraft ground speed $V$, according to

$$
\Delta t=[(u / V) /(1-u / V)] t
$$

Headwind (tailwind) effects imply that the younger (older) vortex has covered a smaller (larger) descent distance at a given observation time. In P2P predictions vortex age is corrected according to

$$
t_{\text {cor }}=t-\Delta t=[(1-2 u / V) /(1-u / V)] t
$$


Another effect arises from axial wind for descending and climbing aircraft. For instance, for descending aircraft the vortex advected by headwind (tailwind) is generated at lower (higher) altitude compared to the generation height within the control plane. The respective height difference $\Delta z$ depends on age difference, flight speed, and glide-slope angle $\varepsilon$, according to

$$
\Delta z=\Delta t V \tan \varepsilon
$$

For example, for an aircraft facing a headwind of $u=-7 \mathrm{~m} / \mathrm{s}$ at an approach speed of $V=70 \mathrm{~m} / \mathrm{s}$ on a glide slope of $3 \mathrm{deg}$, the age difference amounts to more than $-9 \%$ with a corresponding altitude offset of $-33 \mathrm{~m}$ at a nominal vortex age of $100 \mathrm{~s}$. Figure 7 compares lidar measurement results to predictions with and without headwind correction in a case where the aircraft descends on a glide slope of $\varepsilon=3.9 \mathrm{deg}$ with a tailwind of $u^{*} \approx 3$. Clearly, with the tailwind correction predictions reproduce measurements better. At the time of the last measurement, the height difference in the deterministic predictions amounts to $\Delta z^{*}=0.62$.

To ensure that the vortices experience the meteorological conditions prevailing at the modified altitudes, the altitude correction (4) is applied incrementally at every time step. Currently, the correction is switched off when the vortices descend to a height below $z^{*}=0.6$ because the interaction of primary and secondary vortices in ground effect is not yet adapted to the height correction procedure.

\section{Wind Shear}

Simulation and experiment both indicate vigorous and variform influences of wind shear on wake-vortex fate. Wake vortices that interact with a shear layer can experience vortex tilting, separation, and subsequent rebound of vortices, ${ }^{20,21}$ whereupon the vortex with

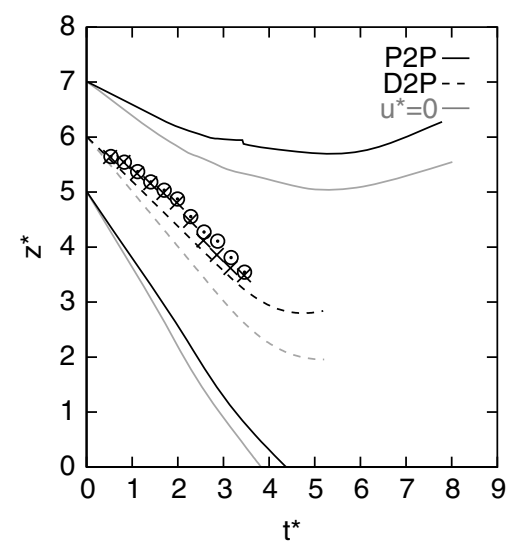

Fig. 7 Measured (symbols) and predicted (lines) descent of wake vortices generated by aircraft on glide slope of $\varepsilon=3.9 \mathrm{deg}$ with tailwind of $u^{*} \approx 3$. Black (gray) lines with (without) tailwind correction. opposite-signed vorticity to the shear layer decays distinctly faster in turbulent shear layers. ${ }^{22}$ These effects are not observed in laminar flows with constant shear, ${ }^{23}$ which provides evidence that the rebound is caused by the vertical gradient of wind shear rather than by the wind shear directly. ${ }^{20,24}$

For real-time wake-vortex prediction different approaches were suggested. References 25 and 26 model shear layers by a system of point vortices that interacts with the primary vortices. The approaches mimic wake-vortex trajectories obtained from solving the two-dimensional Navier-Stokes equations for prototype shear layers. Reference 27 employs the continuous vortex sheet method to model both wake vortices and shear layer. Reference 6 employs the vertical gradient of wind shear to modify wake-vortex circulation or descent speed. ${ }^{3}$

Unfortunately, wake-vortex/shear-layer interaction is extremly sensitive to a number of shear-layer parameters. ${ }^{28}$ Correspondingly, Ref. 9 illustrates that sufficiently precise observations and, in particular, predictions of shear-layer characteristics are hardly feasible. Even with dedicated wind measurement devices it was not always possible to measure shear layers with sufficient accuracy to fully explain the observed vortex behavior. Therefore, deterministic predictions that aim to directly emulate the interaction of wake vortices and the vorticity in the shear layer do not seem to have potential for operational applications. At most, probabilistic approaches can cover shear-layer effects.

A series of measurements from the WakeToul and AWIATORFT1 campaigns suggests that the interaction of wake vortices with shear layers can be categorized by a normalized shear rate according to

$$
s h^{*}=\frac{\partial v}{\partial z} \frac{b_{0}}{w_{0}}
$$

Tilting and stalling or even rebounding vortices (see Fig. 8) are only observed when wake vortices penetrate shear layers with $\left|s h^{*}\right|>1$.

For the parameterization within $\mathrm{P} 2 \mathrm{P}$, the wind velocity difference across a one-vortex-spacing height difference $\Delta v=\partial v / \partial z \cdot b_{0}$, normalized by the vortex descent speed $w_{0}$, can be used as a superimposed shear-induced propagation velocity $v_{\mathrm{sh}}^{*}$. If wake vortices encounter a shear layer with $\left|s h^{*}\right|>1$, the normalized shear velocity widens the envelopes for vortex transport (see Fig. 8) in analogy to the approach proposed for turbulent spreading. ${ }^{4,9}$ Turbulence velocity $q^{*}$ and shear velocity $v_{\mathrm{sh}}^{*}$ are superimposed quadratically to consider the connatural effects of shear and turbulence. An example of this applied to the upper bound of lateral position is

$$
y_{u}^{*}=y^{*}+\int \sqrt{\left(C_{q} q^{*}\right)^{2}+\left(C_{\mathrm{sh}} v_{\mathrm{sh}}^{*}\right)^{2}} \mathrm{~d} t^{*}
$$

where $C_{q}$ and $C_{\mathrm{sh}}$ are constants. Shear-layer uncertainty allowances are applied to both lateral bounds employing $C_{q}=1, C_{\mathrm{sh}}=0.3$ and the upper vertical bound with $C_{q}=0.5, C_{\mathrm{sh}}=0.4$.
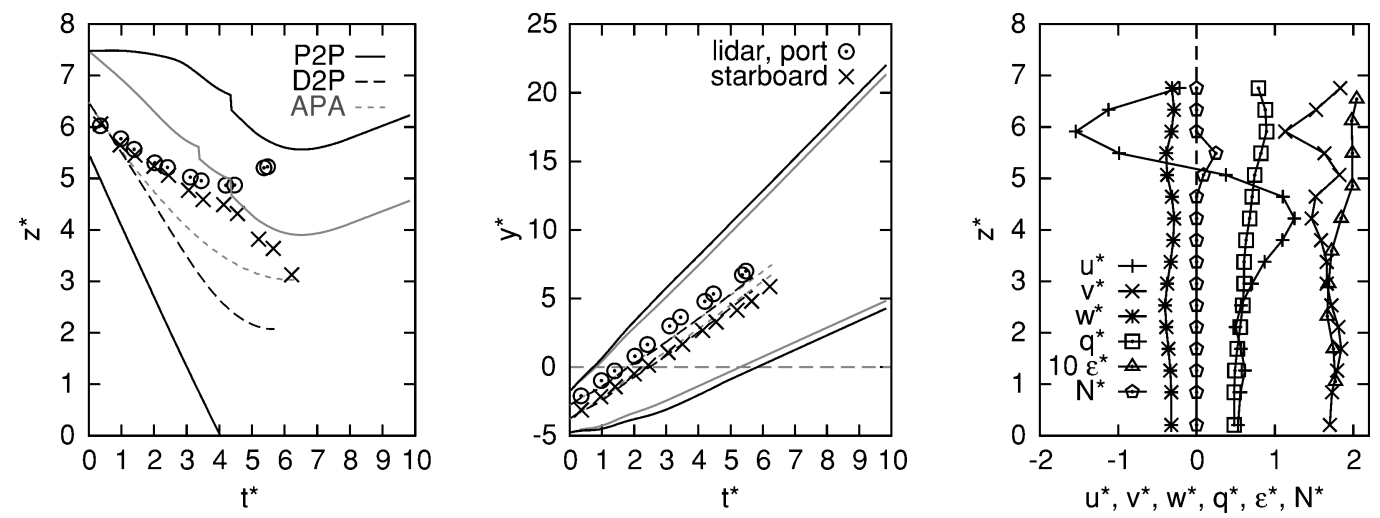

Fig. 8 Measured (symbols) and predicted (lines) evolution of normalized vertical and lateral positions of trailing vortices penetrating a pronounced shear layer (AWIATOR-FT1 flight 1-04). Black dashed lines denote deterministic behavior, black (gray) solid lines the probabilistic envelope with (without) the shear-layer model. Right, vertical profiles of normalized environmental data. 
Some AWIATOR-FT1 cases provide evidence that also axial wind shear $\partial u / \partial z$ can cause similar effects as pure crosswind shear. This can be explained by the fact that wake vortices in the atmospheric boundary layer usually are deformed immediately, whereby the vortices become susceptible to both components of vertical shear. As a consequence, $v_{\mathrm{sh}}^{*}$ employs the magnitude of both vertical windshear components. To prevent from excessive growth of uncertainty allowances driven by turbulence and wind shear, it is assumed that shear-induced propagation velocities can at maximum achieve the magnitude of the current vortex descent speed. Further, a temporal relaxation of $v_{\mathrm{sh}}^{*}$, which considers that shear-layer effects persist beyond the passage of the shear layer, is adopted. Figure 8 illlustrates that the probabilistic envelopes, which in that case are mainly widened because of axial wind shear, successfully enclose the observed vortex rebound.

\section{Assessment of Wake Prediction Skill}

\section{Deterministic Model Performance}

To evaluate the basic performance of the two-phase model, its deterministic version (termed D2P) is employed to predict mean vortex evolutions (see Figs. 1, 4, 8, black dashed lines), which are compared to predictions of the APA ${ }^{8,29}$ (gray dashed lines). For this purpose 24 WakeToul cases and 25 AWIATOR-FT1 cases, which provide complete high-quality vortex and meteorological data, are selected. Mean eddy dissipation rates and Brunt-Väisälä frequencies prevailing during the corresponding overflights are plotted in Fig. 2 with dark gray symbols.

The scoring procedure, which is described in detail in Ref. 30, was already applied to APA and D2P before. ${ }^{9}$ It evaluates the rms deviations of measurement and prediction of the quantities $y^{*}, z^{*}$, and $\Gamma_{5-15}^{*}$ for each overflight. For an individual overflight the scoring is terminated with either the last lidar datum or when predicted circulations go to zero. From the distribution of rms values resulting from the 49 cases, the median and the 90th percentile are used to characterize the performance of the models. Table 1 indicates advantages for D2P regarding lateral and vertical transport ranging from 7 to $38 \%$. The significant advantages regarding circulation evolution result from the good agreement between measured and predicted two-phase decay characteristics.

These results are surprising with respect to the similar performance of APA and D2P achieved in the former scoring test. ${ }^{9}$ Several reasons can contribute to the unequal rating achieved by the models in the different tests. In Ref. 9 deviations of measurement and prediction were strongly affected by inconsistencies resulting from the 2-km separation of the sites for meteorological and wake-vortex data acquisition and by poorer wake-vortex data quality caused by the fully automatic data evaluation procedure applied to the large number of overflights. Moreover, APA was adapted to the database considered in Ref. 9 regarding decay constant $C$ and vortex spacing, whereas in this comparison D2P was adapted to the data regarding decay parameter $T_{2}^{*}$.

Table 2 shows scoring results achieved with D2P based on the complete set of 64 overflights for different sources of crosswind. The comparison with Table 1 indicates a slight improvement of descent predictions, which is juxtaposed to a slight degradation regarding circulation. For lateral transport crosswinds provided by lidar yield clearly superior scoring results compared to sodar crosswinds. Nevertheless, sodar wind data are used in this study by default because lidar does not provide axial wind and wind-shear data.

Table 1 Statistics for normalized differences between deterministic model predictions and selected observations from WakeToul and AWIATOR-FT1 campaigns (49 cases)

\begin{tabular}{llccc}
\hline \hline Model & Averages & $\mathrm{rms} \Delta y^{*}$ & $\mathrm{rms} \Delta z^{*}$ & $\mathrm{rms} \Delta \Gamma^{*}$ \\
\hline APA & Median & 0.917 & 0.563 & 0.219 \\
& 90th perc. & 1.80 & 1.07 & 0.387 \\
D2P & Median & 0.838 & 0.407 & 0.103 \\
& 90th perc. & 1.68 & 0.999 & 0.221 \\
\hline \hline
\end{tabular}

Table 2 Statistics for normalized differences between D2P predictions and 64 observations from WakeToul and AWIATOR-FT1 campaigns for different crosswind measurement devices

\begin{tabular}{lcccc}
\hline \hline Crosswind source & Averages & $\mathrm{rms} \Delta y^{*}$ & $\mathrm{rms} \Delta z^{*}$ & $\mathrm{rms} \Delta \Gamma^{*}$ \\
\hline Lidar & Median & 0.578 & 0.385 & 0.128 \\
& 90th perc. & 1.38 & 0.847 & 0.287 \\
Sodar & Median & 0.962 & 0.385 & 0.128 \\
& 90th perc. & 2.59 & 0.847 & 0.287 \\
\hline \hline
\end{tabular}

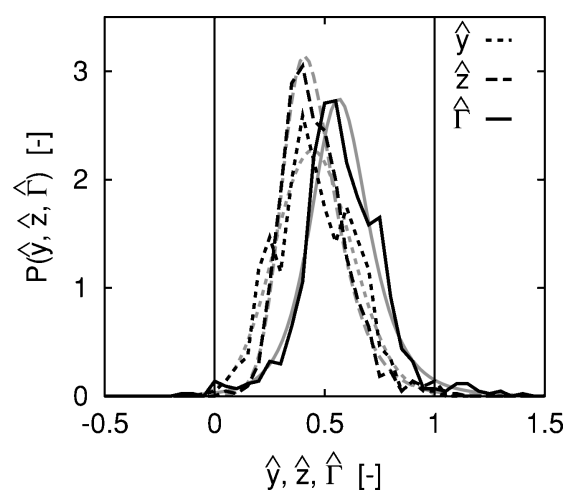

Fig. 9 Probability density distributions of measured lateral position, vertical position, and circulation of wake vortices normalized with respect to the uncertainty bounds predicted by P2P. Values of zero and one denote lower and upper bounds, respectively. Fits of respective unbounded Johnson distributions denoted by gray lines.

\section{Probabilistic Model Performance}

Probabilistic model performance cannot be evaluated based on scoring approaches because increased uncertainty allowances would always improve ratings. Detailed assessment of probabilistic wake prediction skill is achieved by the compilation of probability density distributions (PDD), which relate vortex measurement data to the upper and lower bounds of the predicted uncertainty allowances (see Fig. 9). For this purpose, the value of every single vortex datum is normalized according to

$$
\hat{y}=\left(y_{\text {meas }}-y_{l}\right) /\left(y_{u}-y_{l}\right)
$$

Equation (7), which here is exemplarily expressed for lateral position, assigns a value of one to a vortex measurement situated on the predicted upper bound (index $u$ ) and the value zero to measurements on the lower bound (index $l$ ).

Measurements of the whole vortex evolution-from generation to decay-are included in the statistics. The shown PDD employs data of 49 overflights out of two campaigns, six days, and 872 vortex observations, respectively. Only overflights are used, where vortex and meteorological data were measured completely and with high quality. Therefore, the PDDs should mainly represent the intrinsic variability of vortex evolution and to a lesser extent the uncertainties of the input parameters of P2P. The employed data sample corresponds to the data being used to generate the scoring results in Table 1 .

The selected overflights include many long-lived vortices and complex cases with for example pronounced shear effects. Note that the PDD for vortex descent is skewed because of a few cases with retarded descent caused by shear layers. Also the PDD for circulation is not centered around $\hat{\Gamma}=0.5$ because it is only optimized regarding the relevant upper boundary $\hat{\Gamma}=1$. The lower bound for circulation is irrelevant. All other flanks of the PDDs decline steeply when approaching the probabilistic bounds 0 and 1 , which indicates that the applied uncertainty allowances are close to an optimum. Counting the outliers $(\hat{y}, \hat{z}<0 \vee \hat{y}, \hat{z}>1, \hat{\Gamma}>1)$, the probabilistic $2 \mathrm{P}$ model could predict wake-vortex behavior conservatively in $99.7,99.7$, and $97.9 \%$ of the observations for lateral position, vertical position, and circulation, respectively.

Figure 9 demonstrates further that unbounded Johnson $S_{U}$ distributions (gray lines) fit the PDDs reasonably well. The fits pass 
the Kolmogorov-Smirnov goodness-of-fit test at a significance level 0.05 . Valid PDD fits are likely to provide probability estimates that can be extrapolated beyond the range of the finite number of the so far available experimental measurements. The empirical Johnson $S_{U}$ family of distributions ${ }^{31}$ is based on the transformation of a standard normal variate (deviate) according to

$$
\hat{y}=\varepsilon+\lambda \sinh [(z-\gamma) /(\eta)]
$$

where $\hat{y}$ is the fitted variable, $z$ is a deviate, and $\varepsilon, \lambda, \gamma, \eta$ are parameters of the Johnson $S_{U}$ distribution. An advantage of such a transformation is that estimates of probabilities can be obtained from the standard normal distribution.

\section{Probabilistic and Stochastic Prediction Methods}

An apparent advantage of such like established PDDs is that the probability with which vortex evolution develops within the hitherto predicted bounds can be specified. Quantitative knowledge of wake-vortex prediction skill is required to estimate risk probabilities of reduced wake-vortex spacing systems. Moreover, the knowledge of PDDs, which map predicted vortex behavior to real vortex behavior, establishes new alternatives for wake-vortex prediction methodologies.

\section{Arbitrary Degrees of Probability}

In principle, the approach described in the preceding section allows one to provide the temporal evolution of full PDDs for vortex position and circulation based on a given prediction of upper and lower bounds of the respective quantities. In practice that is predictions of envelopes which constitute arbitrary probabilities can be prescribed. Once the value $\hat{y}$ that represents a selected probability (set by the value of $z$ within the standard normal distribution) is calculated from Eq. (8), the respective vortex property is achieved by inverting of Eq. (7):

$$
y_{\text {prob }}=\hat{y}\left(y_{u}-y_{l}\right)+y_{l}
$$

As an example for the possible model output, Fig. 10 shows the temporal evolution of six selected confidence levels for vortex positions and circulation and the respective lidar measurement data of AWIATOR-FT1 overflight 1-03. Similarly as in Fig. 8, which shows the subsequent overflight 1-04 with almost identical meteoreological conditions, the vortices penetrate a pronounced axial wind shear layer. However, in contrast to Fig. 8, it is now the starboard vortex that rebounds. This indicates that random deformations of the vortices can render either vortex susceptible to axial wind shear. Evidently, this is a situation that cannot be predicted deterministically. As the observed rebound is relatively unlikely, the rebounding vortex quits the $2 \sigma$ envelope $(95.4 \%$ ).

Although the data sample used to fit the PDDs is not yet very large, it, nonetheless, comprises a variety of complex wake-vortex cases. Presumably, the shape of the distributions will not be fundamentally modified by an enlarged data sample. Even though the favorable goodness-of-fit test results support the statistical significance of the underlying data sample, obviously, further high-quality wake-vortex measurement data are needed to corroborate in particular the significance of the tails of the PDDs. The accuracy of the tails is of utmost importance because the tails include extreme, safety relevant deviations from mean wake-vortex behavior.

Given a future large amount of high-quality experimental data, the described method can be further improved regarding two aspects. Currently, the PDDs do not depend on vortex age. As a consequence, the standard deviation of $\Gamma^{*}$, for example, is grossly overestimated at early times (compare Fig. 5). PDDs established for different phases of vortex evolution could clearly improve predictions. Furthermore, currently the PDDs for $\hat{y}, \hat{z}, \hat{\Gamma}$ are independent. In reality there is, for instance, a positive correlation between vortex strength and descent. That is, long-lived vortices descend farther than short-lived vortices. Figure 11 depicts a three-dimensional joint probability density distribution (JPDD), which is derived from the measurement data of the 49 overflights. The JPDD clearly features the correlation between $\hat{\Gamma}$ and $\hat{z}$ in the $(\hat{z}, \hat{\Gamma})$ plane (maximum at $\hat{z}=0.4, \hat{\Gamma}=0.6$ ). The absolute maximum at $\hat{\Gamma}=0.7$ and $\hat{y}, \hat{z}=0.4$, illustrated by the central jewel-shaped isosurface, roughly agrees with the independent maxima plotted in Fig. 9. However, the data sample still is much too small to provide a sufficiently smooth JPDD, which could allow for joint probabilistic wake-vortex prediction.

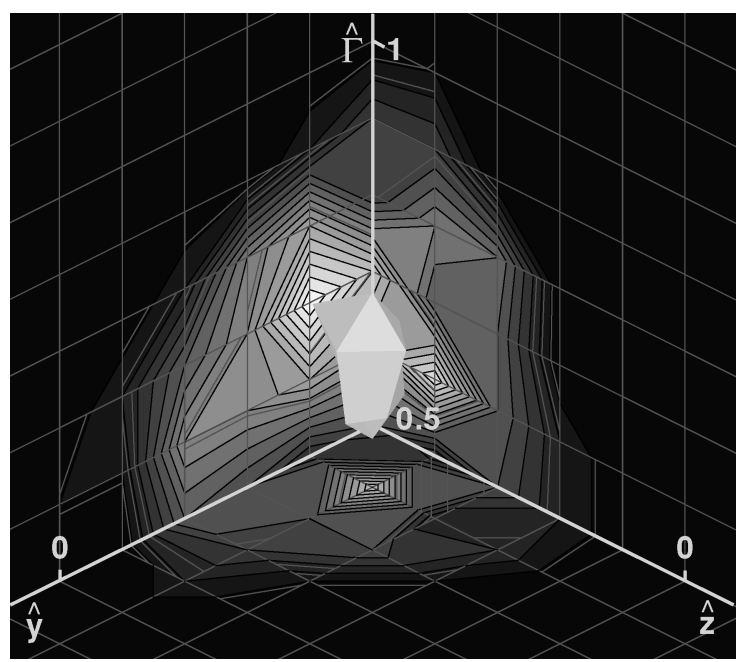

Fig. 11 Three-dimensional joint probability density distribution of measured lateral position, vertical position, and circulation of wake vortices normalized with respect to the uncertainty bounds predicted by P2P. Values of zero and one denote lower and upper bounds, respectively. Isolines represent JPDD values in the three displayed planes; centric isosurface surrounds location of absolute maximum of JPDD.
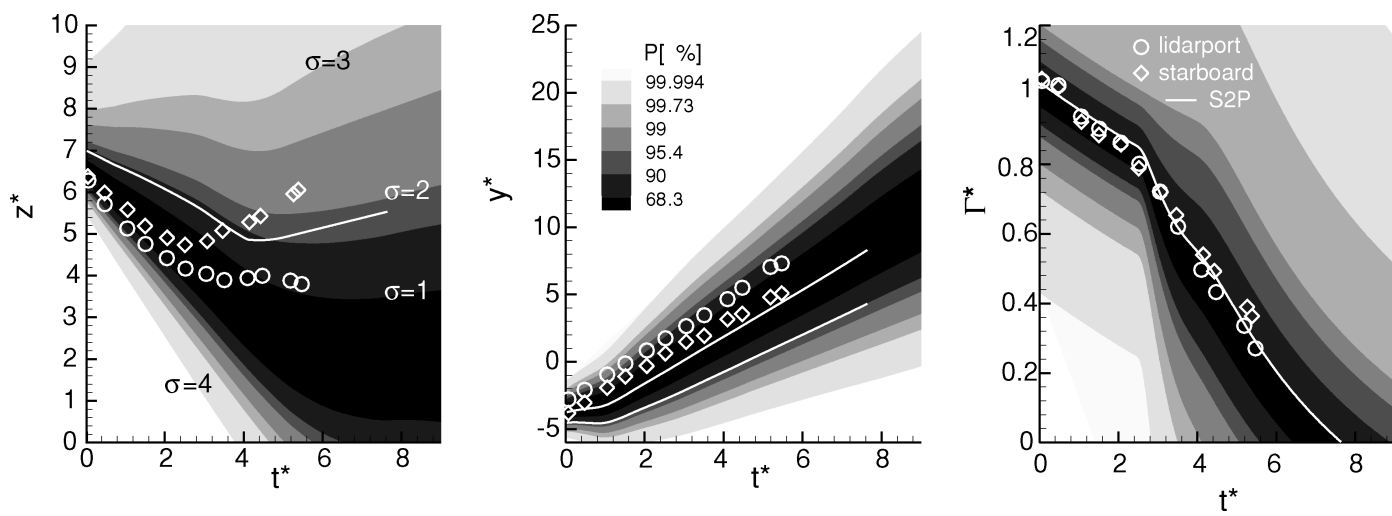

Fig. 10 Prediction of envelopes with six different confidence levels and respective stochastic prediction (S2P) of vertical and lateral position as well as circulation of wake vortices. 


\section{Stochastic Prediction Method}

An alternative for wake-vortex predictions based on the derived Johnson $S_{U}$ distributions is a stochastic approach. For example, in a Monte Carlo simulation environment, which is used to perform safety analyses for new aircraft or new procedures, vortex prediction constitutes one of the key elements that should be varied stochastically. So far, probabilistic safety assessment models like the wake-vortex induced risk assessment model WAVIR ${ }^{32}$ still employ a deterministic wake-vortex model and merely varies its input.

The stochastic two-phase model ( $\mathrm{S} 2 \mathrm{P}$ ) provides random realizations within the probabilistic $S_{U}$ distributions. Normally distributed variates are transformed via Eq. (8) into $S_{U}$ variates, which are mapped on stochastic dimensional vortex properties according to Eq. (9). Four different variates are employed for vertical position, lateral position of port and starboard vortex, and circulation, respectively. White lines in Fig. 10 illustrate one possible realization of the S2P model.

\section{Conclusions}

The P2P model has been applied to high-quality data of two measurement campaigns accomplished at Tarbes Airport, France. The pursued measurement strategy and the lidar data-processing technique enabled observations from vortex generation to a progressed state of decay. More than 40 overflights clearly supply evidence of the two-phase circulation decay parameterized by P2P. These findings should be suitable to contribute to the conclusion of the long-time controversial debate on vortex decay characteristics. ${ }^{1,17}$

With the parameterizations of the effects of axial wind and wind shear, the P2P model completely considers all relevant environmental parameters that could be identified so far. While the parameterization of axial wind and glide-slope angle correct for vortex age and descent height, wind-shear effects enlarge probabilistic envelopes. From an operational point of view, neither predictions nor measurements of vertical wind profiles can be accurate and representative enough to allow for deterministic forecasts of wind-shear effects. It is found that also axial wind shear can cause similar rebounds as crosswind shear, probably because rapid vortex deformation renders vortices susceptible to both components of vertical shear. Examples demonstrate that the new model elements are adequate and improve wake prediction skill.

In a scoring procedure, deterministic model predictions of D2P and $\mathrm{APA}^{8}$ are compared. Slight advantages of D2P regarding lateral and vertical transport are juxtaposed to D2P's clearly superior circulation decay predictions, which reflect the good agreement between measured and predicted two-phase decay characteristics.

A method for the assessment of probabilistic model performance is suggested. For this purpose probability density distributions (PDD), which relate vortex measurement data to the bounds of the predicted uncertainty allowances, are compiled. Based on 49 overflights, $99.7,99.7$, and $97.9 \%$ of the observations for lateral position, vertical position, and circulation, respectively, are predicted conservatively. The devised procedure allows assigning confidence intervals to the hitherto predicted bounds. Moreover, predictions with arbitrary degrees of probability are enabled. Defined confidence intervals are needed to consistently combine probabilistic wake-vortex prediction with other probabilistic components of a wake-vortex advisory system like, for instance, a model for aircraft adherence to glide path. Combination of confidence intervals of all elements of a wake-vortex advisory system allow the performance of a safety analysis of the complete system architecture.

Obviously, the relatively small data sample comprising 49 overflights does not yet provide the comprehensive statistics needed for reliable prediction of vortex-parameter envelopes with arbitrary degrees of probability. However, the developed methodology allows the continuous enhancement of P2P performance based on future high-quality measurement data being used to augment the statistical basis of the PDDs. In other words, the suggested approach allows the training of the model based on observations. Herewith, the model output does not only gain probabilistic substance, but it is also modified concerning the predicted mean, and it comprises higher moments as variance, skewness, and kurtosis. It is assumed that the devised assessment method and training process are also applicable to vortex evolution in ground proximity, which so far has not been investigated in detail and is subject of ongoing work.

\section{Acknowledgments}

The essential contributions of all of the teams during the WakeToul and AWIATOR-FT1 trials and the subsequent data processing are gratefully acknowledged. The WakeToul campaign has been carried out in the framework of the European research program C-Wake under contract No. GRD1-1999-10332; the AWIATOR-FT1 deployment has been performed under contract No. G4RD-2002-00836. Both trials have been cofunded by the DLR Project Wirbelschleppe.

\section{References}

${ }^{1}$ Gerz, T., Holzäpfel, F., and Darracq, D., "Commercial Aircraft Wake Vortices," Progress in Aerospace Sciences, Vol. 38, No. 3, 2002, pp. 181-208.

${ }^{2}$ Hemm, R., Shapiro, G., Lee, D. A., Gribko, J., and Glaser, B., "Benefit Estimates of Terminal Area Productivity Program Technologies," NASA/CR-1999-208989, Jan. 1999.

${ }^{3}$ Gerz, T., Holzäpfel, F., Bryant, W., Köpp, F., Frech, M., Tafferner, A., and Winckelmans, G., "Research Towards a Wake-Vortex Advisory System for Optimal Aircraft Spacing," Comptes Rendus Physique, Vol. 6, No. 4-5, 2005, pp. 501-523.

${ }^{4}$ Holzäpfel, F., "Probabilistic Two-Phase Wake Vortex Decay and Transport Model," Journal of Aircraft, Vol. 40, No. 2, 2003, pp. 323-331.

${ }^{5}$ Sarpkaya, T., "New Model for Vortex Decay in the Atmosphere," Journal of Aircraft, Vol. 37, No. 1, 2000, pp. 53-61.

${ }^{6}$ Jackson, W., Yaras, M., Harvey, J., Winckelmans, G., Fournier, G., and Belotserkovsky, A., "Wake Vortex Prediction-An Overview," Transport Canada, Rept. TP 13629E, Montreal, March 2001.

${ }^{7}$ Robins, R. E., Delisi, D. P., and Greene, G. C., "Algorithm for Prediction of Trailing Vortex Evolution," Journal of Aircraft, Vol. 38, No. 5, 2001, pp. 911-917.

${ }^{8}$ Sarpkaya, T., Robins, R. E., and Delisi, D. P., "Wake-Vortex EddyDissipation Model Predictions Compared with Observations," Journal of Aircraft, Vol. 38, No. 4, 2001, pp. 687-692.

${ }^{9}$ Holzäpfel, F., and Robins, R. E., "Probabilistic Two-Phase Aircraft Wake-Vortex Model: Application and Assessment," Journal of Aircraft, Vol. 41, No. 5, 2004, pp. 1117-1126

${ }^{10}$ Holzäpfel, F., Gerz, T., Köpp, F., Stumpf, E., Harris, M., Young, R. I., and Dolfi-Bouteyre, A., "Strategies for Circulation Evaluation of Aircraft Wake Vortices Measured by Lidar," Journal of Atmospheric and Oceanic Technology, Vol. 20, No. 8, 2003, pp. 1183-1195.

${ }^{11}$ Köpp, F., Rahm, S., and Smalikho, I., "Characterization of Aircraft Wake Vortices by $2-S \mu \mathrm{m}$ Pulsed Doppler Lidar," Journal of Atmospheric and Oceanic Technology, Vol. 21, No. 2, 2004, pp. 194-206.

${ }^{12}$ Köpp, F., Rahm, S., Smalikho, S., Dolfi, A., Cariou, J.-P., Harris, M., and Young, R. I., "Comparison of Wake-Vortex Parameters Measured by Pulsed and Continuous-Wave Lidars," Journal of Aircraft, Vol. 42, No. 4, 2005, pp. 916-923.

${ }^{13}$ Smalikho, I., Köpp, F., and Rahm, S., "Measurement of Atmospheric Turbulence by 2- $\mu \mathrm{m}$ Doppler Lidar,' Inst. für Physik der Atmosphäre, DLR, Rept. 200, Oberpfaffenhofen, Germany, Aug. 2004; Journal of Atmospheric and Oceanic Technology (to be published).

${ }^{14}$ Proctor, F. H., and Switzer, G. F., "Numerical Simulation of Aircraft Trailing Vortices," Proceedings of the 9th Conference on Aviation, Range and Aerospace Meteorology, Paper 7.12, American Meteorological Society, Orlando, FL, 2000, pp. 511-516.

${ }^{15}$ Holzäpfel, F., Gerz, T., and Baumann, R., "The Turbulent Decay of Trailing Vortex Pairs in Stably Stratified Environments," Aerospace Science and Technology, Vol. 5, No. 2, 2001, pp. 95-108.

${ }^{16}$ Holzäpfel, F., Hofbauer, T., Darracq, D., Moet, H., Garnier, F., and Ferreira Gago, C., "Analysis of Wake Vortex Decay Mechanisms in the Atmosphere," Aerospace Science and Technology, Vol. 7, No. 4, 2003, pp. 263-275.

${ }^{17}$ Spalart, P. R., "Airplane Trailing Vortices," Annual Review of Fluid Mechanics, Vol. 30, 1998, pp. 107-138.

${ }^{18}$ Loucel, R. E., and Crouch, J. D., "Flight-Simulator Study of Airplane Encounters with Perturbed Trailing Vortices," AIAA Paper 2004-1074, Jan. 2004.

${ }^{19}$ Frech, M., and Zinner, T., "Concept of Wake Vortex Behavior Classes," Journal of Aircraft, Vol. 41, No. 3, 2004, pp. 564-570.

${ }^{20}$ Proctor, F. H., Hinton, D. A., Han, J., Schowalter, D. G., and Lin, Y.-L., "Two-Dimensional Wake Vortex Simulations in the Atmosphere: Preliminary Sensitivity Studies," AIAA Paper 97-0056, Jan. 1997.

${ }^{21}$ Darracq, D., Moet, H., and Corjon, A., "Effects of Crosswind Shear and Atmospheric Stratification on Aircraft Trailing Vortices," AIAA Paper 99-0985, Jan. 1999 
${ }^{22}$ Hofbauer, T., and Gerz, T., "Shear-Layer Effects on the Dynamics of a Counter-Rotating Vortex Pair," AIAA Paper 2000-0758, Jan. 2000.

${ }^{23}$ Hofbauer, T., "Numerische Untersuchungen zum Einfluss von Windscherung und Turbulenz auf Flugzeugwirbelschleppen," DLR, Forschungsbericht 2003-01, Inst. für Physik der Atmosphäre, Oberpfaffenhofen, Germany, 2003.

${ }^{24}$ Burnham, D. C., "Effect of Ground Wind Shear on Aircraft Trailing Vortices," AIAA Journal, Vol. 10, No. 8, 1972, pp. 1114-1115.

${ }^{25}$ Zheng, Z. C., and Baek, K., "Inviscid Interactions Between Wake Vortices and Shear Layers," Journal of Aircraft, Vol. 36, No. 2, 1999, pp. 477-480.

${ }^{26}$ Meleshko, V. V., Gurzhi, A. A., Dörnbrack, A., Gerz, T., Holzäpfel, F., and Hofbauer, T., "Interaction of Two-Dimensional Trailing Vortex Pair with a Shear Layer," International Applied Mechanics, Vol. 37, No. 7, 2001, pp. 948-957.
${ }^{27}$ Mokry, M., "Numerical Simulation of Aircraft Trailing Vortices Interacting with Ambient Shear or Ground," Journal of Aircraft, Vol. 38, No. 4, 2001, pp. 636-643.

${ }^{28}$ Hofbauer, T., and Holzäpfel, F., "Behavior of Aircraft Wake Vortices Subjected to Wind Shear," AIAA Paper 2003-3813, June 2003.

${ }^{29}$ Robins, R. E., and Delisi, D. P., "NWRA AVOSS Wake Vortex Prediction Algorithm Version 3.1.1," NASA/CR-2002-211746, June 2002.

${ }^{30}$ Robins, R. E., and Delisi, D. P., "Wake Vortex Algorithm Scoring Results," NASA/CR-2002-211745, June 2002.

${ }^{31}$ Hahn, G. J., and Shapiro, S. S., Statistical Models in Engineering, Wiley, New York, 1967, pp. 198-220.

${ }^{32}$ de Bruin, A. C., Speijker, L. J. P., Moet, H., Krag, B., Luckner, R., and Mason, S., "S-Wake-Assessment of Wake Vortex Safety," Publishable Summary Report, National Aerospace Lab., NLR-TP-2003-243, Amsterdam, May 2003. 